\section{Criterios de localización de estaciones intermodales: propuesta para el Área Metropolitana de Caracas}

Marjely Caneva Rodríguez

Coordinación de Estudios Urbanos, Universidad Simón Bolívar, Venezuela.

\section{Josefina Flórez Díaz}

Departamento de Planificación Urbana, Universidad Simón Bolívar, Venezuela.

Recibido: 30 de octubre de 2017. Aceptado: 16 de mayo de 2018

\title{
Resumen
}

El Área Metropolitana de Caracas (AMC) posee sistemas de transporte público que operan de manera independiente, generando duplicación de rutas, áreas informales de trasbordo, aumento de los tiempos de viajes, conflictos con modos no motorizados y deterioro del espacio público, entre otros. En el marco de la movilidad sostenible, las estaciones intermodales tienen el potencial de facilitar la integración física de los modos de transporte y contribuir a solucionar la problemática existente. A pesar de esto, no hay estudios disponibles que analicen los criterios de localización de estaciones intermodales en el AMC. Con base en el estado del arte, la aplicación de una encuesta semi-estructurada dirigida a expertos y el desarrollo de un panel de profesionales, se definen, clasifican y jerarquizan criterios de localización de estaciones intermodales aplicables al área de estudio y se diseña una propuesta de localización para la ciudad. Se concluye que el AMC se beneficiaría positivamente con la implantación de estos equipamientos y que los criterios identificados son pertinentes y aplicables al área de estudio. Además, se propone que las estaciones intermodales estén vinculadas al sistema Metro y localizadas donde se maximice el acceso a diferentes modos de transporte, en sintonía con políticas de movilidad actualizadas.

\section{Location criteria for intermodal transit stations: proposal for the Metropolitan Area of Caracas}

\footnotetext{
Abstract

The public transport systems of the Metropolitan Area of Caracas (AMC) operate independently, generating duplication of routes, informal transfer areas, increase travel times, conflicts with non-motorized modes and deterioration of the public space,
}

Palabras claves

Intermodalidad Multimodalidad Transferencia modal Estaciones intermodales Caracas

Palavras-chave

Intermodalidade Multimodalidade Transferência modal Estações intermodais Caracas

Keywords

Intermodality Multimodality Modal transfer Intermodal stations Caracas 
among others. Within the framework of sustainable mobility, intermodal stations have the potential to facilitate the physical integration of transport modes and contribute to the solution of the existing problems. However, there are no available studies that analyze the location criteria of intermodal stations in the AMC. Based on the state of the art, the application of a semi-structured survey directed to experts and the development of a workshop of professionals, criteria for the location of intermodal stations applicable to the AMC are defined, classified and ranked. Besides, a proposal for the location of these stations in the city is designed. It is concluded that the AMC would benefit from the implementation of these stations and that the identified criteria are relevant and applicable to the study area. In addition, it is proposed that the intermodal stations are linked to the Metro system and located where access to different modes of transport is maximized, in line with updated mobility policies.

\section{Introducción}

El modelo de desarrollo urbano que ha prevalecido en el Área Metropolitana de Caracas (AMC), su relación con otras subunidades de la Región Metropolitana (RMC) y otros aspectos económicos y políticos, han definido patrones de movilidad pocos sostenibles en la ciudad (IERU, 2008).

A pesar de que la mayor parte de los viajes se realizan en transporte público (INMETRA, 2005), algunas políticas del Estado han favorecido el uso del automóvil (Lizarraga, 2012), tales como mantener los precios bajos de la gasolina y la creación del programa Venezuela Móvil que hasta el 2008, exoneraba del pago del Impuesto al Valor Agregado (IVA) a las operaciones de importación, venta interna y adquisición de vehículos y componentes asociados que cumplieran con las condiciones de producción y comercialización definidas en el programa (Decreto No. 3692 del Programa Venezuela Móvil, 2005).

A eso se suman las deficiencias en el sistema de transporte público asociadas a la inadecuada integración de los modos que operan en la ciudad y que traen como consecuencia la realización de recorridos innecesarios en transporte superficial, muchos de ellos hacia el centro de la ciudad, congestión, aumentos de los costos y tiempos de viaje, deterioro del espacio urbano, entre otros (Barriga Dall'Orto - SOMELCA, 2000; Lizarraga, 2012).

Muchas de las condiciones mencionadas han sido un factor común en diferentes ciudades latinoamericanas. Dentro de ellas, Bogotá y Curitiba han sido reconocidas internacionalmente por la aplicación de estrategias y políticas de transporte que favorecen la integración modal y la movilidad sostenible (Chaparro, 2002; Mello y Rojas, 2005; UIAM, 2011).

El concepto de intermodalidad se refiere a la adecuada integración institucional, operacional y física, entre más de un modo de transporte para facilitar los viajes puerta a puerta de bienes o personas y optimizar el aprovechamiento de las ventajas técnicas de cada uno (Goncalves, 1990; Pitsiava-Latinopoulou y Iordanopoulos, 2012). En estos términos, la intermodalidad constituye una pieza clave en la movilidad sostenible.

Particularmente, para la integración física de los sistemas de transporte, resultan fundamentales las estaciones intermodales, ya que facilitan la transferencia entre los diferentes modos (Goncalves, 1990). Considerando que el trasbordo suele identificarse como una de las desventajas comparativas del transporte público respecto al vehículo particular, la adecuada planificación y diseño de estas infraestructuras es de gran relevancia (Pitsiava-Latinopoulou y Iordanopoulos, 2012). 
Particularmente, su localización debe guiarse por una serie de criterios que, entre otros aspectos, garantice que su desarrollo no influya negativamente en la dinámica urbana, minimice el impacto ambiental y maximice el aprovechamiento de las ventajas tecnológicas de cada modo de transporte (Caneva, 2012). A pesar de la importancia de estas estaciones intermodales y la necesidad de contar con criterios que orienten su localización en la ciudad, se observa que no existen estudios disponibles dirigidos a su definición en el AMC. Investigaciones relacionadas se orientan a la definición la localización de terminales interurbanas para sistemas de autobuses (Ocaña y Gómez, 2016) y en la determinación de los requerimientos para la localización de estacionamientos disuasorios e intercambiadores en la subunidad Altos Mirandinos, en el este de la Región Metropolitana de Caracas (Rojas y Solórzano, 2014).

El objetivo de este estudio es definir y clasificar criterios de localización de estaciones intermodales que sean aplicables al AMC. Los resultados contribuirán en el diseño de políticas y lineamientos dirigidos a promover la movilidad sostenible y favorecer los viajes en transporte público.

Para lograr el objetivo planteado, con base en la revisión del estado del arte se identifican y clasifican criterios generales de localización, los cuales son evaluados a través de una encuesta semi-estructurada aplicada a un grupo de expertos internacionales con conocimiento sobre el sistema de transporte en el AMC y del desarrollo de un panel de profesionales venezolanos del transporte. Los criterios son agrupados en tres ámbitos de análisis: normativo, metropolitano y local. Paralelamente, se levanta y analiza información sobre las características urbanas y del sistema de transporte del AMC a fin de aplicar los criterios y hacer una propuesta de localización de las estaciones acorde con los requerimientos de esta ciudad.

Este artículo se estructura en siete apartados, incluyendo la introducción y conclusiones. En el segundo apartado se caracteriza el área de estudio. Seguidamente, con base en la literatura consultada, se exponen los conceptos asociados a las estaciones intermodales y se indican las etapas del proceso de implantación. Posteriormente se identifican los criterios y se clasifican según su ámbito. En el quinto apartado se describe el proceso de evaluación de los criterios identificados y se presentan los resultados de la investigación. Finalmente, se presenta la discusión y análisis de resultados.

\section{Caracterización y problemática del Área Metropolitana de Caracas}

El Área Metropolitana de Caracas (AMC), junto con las subunidades Guarenas-Guatire, Los Valles del Tuy, Altos Mirandinos y Litoral Vargas, conforman la Región Metropolitana de Caracas (RMC) (Barrios, 2002). Particularmente, el AMC se integra por los municipios Libertador, Chacao, Sucre, Baruta y El Hatillo que, para el año 2011, reunían una población aproximada de 2.900 millones de habitantes, concentrando más de la mitad de la población total de la RMC (INE, 2011).

En sus etapas iniciales el desarrollo urbano del AMC se caracterizó por el aprovechamiento de los terrenos de menores pendientes y su expansión estuvo limitada por la barrera natural definida por el Río Guaire. Entre 1950 y 1971 se registró un fuerte período de crecimiento asociado, en parte, a las medidas planteadas en el Plan Regulador de Caracas del año 1951 (Llanos y Martínez, 2014). Durante este período se destaca el desarrollo de urbanizaciones aisladas de grupos de ingresos medios y de asentamientos no planificados en las colinas que bordean el valle, habitados por familias de bajos ingresos (Flórez, 1998). Adicionalmente, el desarrollo de la red vial alcanzado en esa etapa sirvió como eje de expansión y densificación urbana (Flórez, 2000). 


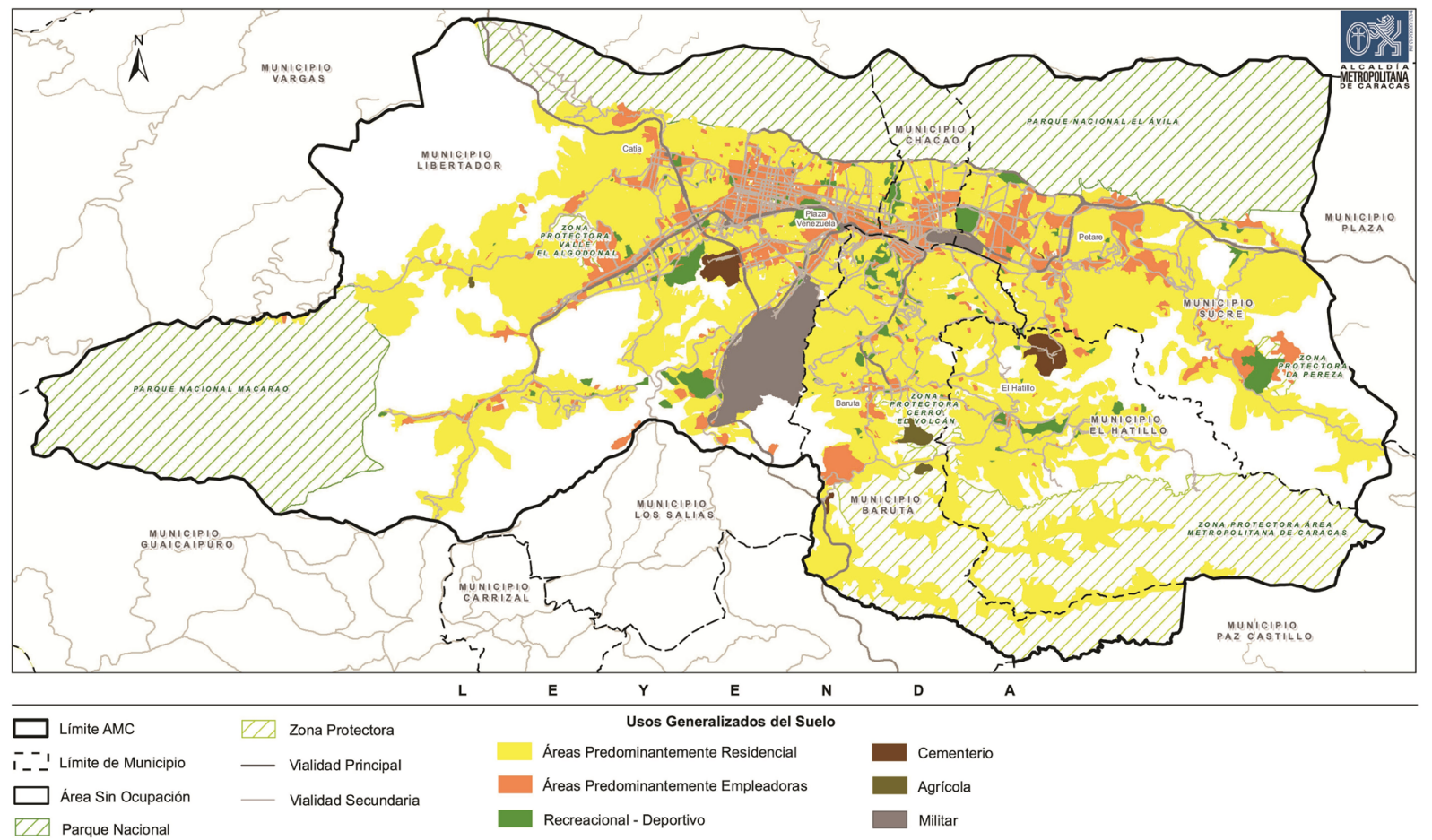

Este proceso de expansión definió la actual estructura del AMC, que se caracteriza por ser una conurbación entre el centro fundacional de la ciudad de Caracas y los centros de menor jerarquía de Chacao, Baruta, Sucre y El Hatillo. Como se observa en la Figura 1, Figura 1. Plano de usos del suelo del AMC. Fuente: Alcaldía en los centros de primer orden se concentran las principales actividades empleadoras, comerciales y de servicios, no solo del AMC sino también del resto de la RMC, mientras que en las zonas periféricas del AMC predominan usos residenciales (Urdaneta, 2013).

Específicamente, se distingue una zona de mayor diversidad en un eje en sentido Este - Oeste localizado en el norte de la ciudad, que concentra las mayores densidades de empleo y servicios. Esta área se extiende longitudinalmente a lo largo del valle desde el Casco Central de Caracas, pasando por el Municipio Chacao hasta el sector Petare - La Urbina. Por otra parte, se destaca un eje de menor jerarquía, correspondiente al sector Valle - Coche y la zona de Catia, donde se desarrollan con menor intensidad, actividades comerciales y de servicios. Dentro de los usos residenciales, destacan, por un lado, los desarrollos informales en los municipios Libertador y Sucre, que poseen las mayores densidades poblacionales y, por otro lado, las viviendas unifamiliares de baja densidad en el sector Sureste de la ciudad, en los municipios Baruta y El Hatillo.

La red vial del AMC está compuesta por vías de alta capacidad que se conectan con el sistema carretero regional y nacional y se complementa con vías secundarias que permiten el acceso a los diferentes usos del suelo. En general, el sistema favorece las conexiones Este - Oeste mientras que los enlaces Norte - Sur se ven limitados, debido en parte, a la existencia de barreras naturales y artificiales como el Río Guaire y la autopista Francisco Fajardo.

El sistema de transporte público se estructura alrededor del Metro, que presenta una línea troncal que favorece las conexiones Este - Oeste a la cual convergen una serie de líneas transversales alimentadoras (IERU, 2008). Adicionalmente, conectados al Metro de Caracas se encuentran: el Metro Los Teques, que comunica el AMC con la ciudad de los Teques ubicada en la subunidad Altos Mirandinos, al oeste de la RMC; y el sistema ferroviario Ezequiel Zamora, que conecta con la subunidad Los Valles del Tuy, al sur 
del AMC. Actualmente se encuentra en construcción la línea superficial de Metro que conectará con Guatire, en la subunidad Guarenas - Guatire en el este de la RMC.

La ciudad cuenta además con dos sistemas de transporte por cable (Metrocables) en los barrios de San Agustín del Sur y Mariches y un sistema de tren ligero elevado (automatic people mover) llamado Cabletren en Petare, que conectan con las estaciones de Metro y sirven a barrios pobres localizados en zonas de altas pendientes. Las tecnologías alternativas de transporte público por cable se han usado en algunas ciudades de América Latina, como Medellín y Río de Janeiro, además de Caracas, para atender población de bajos ingresos localizados en zonas de topografía irregular y con una configuración informal del espacio común (Gonçalves y Bandeira, 2017).

Por su parte, el sistema superficial se compone por el Metrobús, BusCaracas (sistema BRT), TransMetrópoli y un gran número de rutas urbanas y suburbanas, muchas de las cuales sirven como alimentadoras al sistema Metro. Los vehículos característicos de estas rutas son autobuses, minibuses y rústicos (IERU, 2008).

De los mencionados anteriormente, los sistemas Metro de Caracas, Metro de Los Teques, Metrobús, Metrocable, BusCaracas y Cabletren, poseen integración institucional y cuentan con ciertos niveles de integración física y operacional que constituyen oportunidades para el desarrollo de estaciones intermodales. Se destacan además la estación terminal La Rinconada y la estación Caracas Libertador Simón Bolívar, ubicadas al sur del AMC y que son estaciones terminales para los sistemas Metro de Caracas y Ferrocarril Ezequiel Zamora, respectivamente. Gracias a la proximidad de estas estaciones, se facilita la integración física entre ambos sistemas de transporte, aun cuando no poseen integración institucional ni operacional.

Con relación a los patrones de movilidad, la única encuesta Origen-Destino (O-D) del AMC disponible es del año 2005. Con base en los resultados de la encuesta el principal motivo de viaje, después del hogar, es el empleo con un 25\% del total de los viajes realizados. En consecuencia, los destinos de los viajes en las horas pico de la mañana son las zonas empleadoras ubicadas a lo largo del eje central de la ciudad y en las tardes en el sentido contrario. A estos flujos de viajes se les suman aquellos provenientes de otras subunidades de la RMC, cuya población también se encuentra empleada o realiza estudios en el AMC. Con relación al reparto modal, para el año 2005 el 57\% de los viajes eran realizados en transporte público, el $24 \%$ en vehículo particular y el $23 \%$ caminando (INMETRA, 2005).

La problemática del transporte del AMC es compleja y se ve afectada por múltiples elementos del sistema urbano. Entre ellos destacan, la distribución de usos del suelo, las densidades de población y empleo, los patrones de movilidad y las múltiples deficiencias en la red vial y sistemas de transporte público (IERU, 2008).

La distribución de los usos del suelo trae como consecuencia que las necesidades de empleo y servicios de la población se satisfagan principalmente dentro de un área reducida en el eje central de la ciudad. De esta manera, se genera una relación de dependencia que promueve la generación de viajes desde las zonas predominantemente residenciales y contribuye a la saturación del sistema de transporte urbano durante las horas pico del día. Además, considerando que la influencia del eje productivo también afecta a otras subunidades de la RMC, existe una cantidad adicional de viajes interurbanos que incrementan el flujo vehicular generando congestión en las principales vías de acceso al AMC en dichos horarios.

La baja calidad de la infraestructura vial contribuye con los altos niveles de congestión durante las horas pico de la mañana y de la tarde. A raíz de las limitaciones en 
las conexiones Norte - Sur, los usuarios que recorren estas rutas deben utilizar la red vial principal para completar sus viajes, aumentando los flujos vehiculares en las principales autopistas y avenidas y saturando el sistema. Esta situación es acentuada por la incompatibilidad entre la geometría y la función de varias de las vías de la ciudad (IERU, 2008; Lizarraga, 2012), ya que se observan vías de baja capacidad cumpliendo función de colectoras o arteriales para lo cual no fueron diseñadas.

El sistema de transporte público del AMC no se encuentra integrado en su totalidad, y se destacan como principales excepciones los sistemas asociados al Metro de Caracas indicados anteriormente. Particularmente, gran parte del sistema superficial es ofrecido mayormente por el sector privado y se caracteriza por presentar "altos rasgos de informalidad" (Banco de Desarrollo de América Latina, 2011:261) ya que responde de forma no planificada a la demanda producida en cada sector de la ciudad. Esto conlleva a la creación de recorridos innecesarios, duplicación de rutas debido a los intereses competitivos de los operadores y a la generalizada deficiencia de la oferta (Mundó, 2002). Las tarifas en rutas superficiales son independientes a las del sistema Metro y se fijan mediante acuerdos entre los operadores y entidades públicas, donde los primeros tienen mayor influencia en la toma de decisiones (Banco de Desarrollo de América Latina, 2011). Las pocas formas de integración física del sistema superficial son espontáneas y desorganizadas alrededor de las principales estaciones del Metro de Caracas. Ya que estas no son soportadas por una infraestructura adecuada, se generan problemas con modos no motorizados, se interrumpe el flujo vehicular en la vialidad, se aumenta el riesgo de accidentes y se deteriora considerablemente el espacio público (Banco de Desarrollo de América Latina, 2011; IERU, 2008).

La problemática de transporte y movilidad del AMC no ha contado con respuestas tangibles para lograr la integración entre planificación del transporte y usos del suelo y para promover la movilidad sostenible a través de la integración de los modos de transporte (Banco de Desarrollo de América Latina, 2011). Es por esto que el estudio de la localización de estaciones intermodales en el AMC constituye un importante aporte para el cumplimiento de dichos objetivos.

\section{Las estaciones intermodales}

\section{El rol de las estaciones intermodales en la promoción de la movilidad sostenible}

De acuerdo con lo establecido en la conferencia de la ONU sobre Medio Ambiente y Desarrollo (Cumbre de la Tierra) en el año 1992, el desarrollo sostenible debe incluir todos los sectores de la actividad humana, entre ellos el transporte y la movilidad (OECD, 2002). La Comisión de Comunidades Europeas (2000), define la movilidad sostenible como:

un sistema y unas pautas de desplazamiento que proporcionan los medios y las oportunidades para satisfacer las necesidades económicas, ambientales y sociales de manera eficiente y equitativa, al mismo tiempo que minimiza los impactos adversos evitables o innecesarios y sus costes asociados, en escalas espaciales y temporales relevantes.

Entre los objetivos de la movilidad sostenible planteados durante dicha conferencia se encuentran: practicar la planificación conjunta del territorio y del transporte; promover el uso del transporte público de gran capacidad y los modos no motorizados; alcanzar una gestión eficiente de estos sistemas de transporte y conservar adecuadamente la infraestructura asociada (OECD, 2002). Adicionalmente, se definen medidas dirigidas a 
lograr la movilidad sostenible y el cumplimiento de los objetivos. Una de estas medidas es promover la intermodalidad en los sistemas de transporte, definida como un proceso en el cual funcionan de forma integrada y coordinada, más de un modo de transporte para movilizar a los usuarios desde un punto de origen hasta un punto de destino. Este proceso debe resultar coherente, eficaz, energética y socialmente económico (Burckhart, 2007; Goncalves, 1990). La necesidad de integración de los distintos modos de transporte es particularmente relevante en las grandes ciudades donde coexisten redes multimodales de transporte público y presentan altos niveles de congestión (Leclerc, 2002; Pitsiava-Latinopoulou y Iordanopoulos, 2012). Es importante resaltar que la intermodalidad es solo un componente de la sustentabilidad y, por tanto, no posee los atributos necesarios para alcanzar por sí sola la sustentabilidad (Szyliowicz, 2003).

La intermodalidad comprende tres tipos de integración: institucional, operacional y física (Goncalves, 1990; Olvera et al., 2015). La integración institucional se refiere al trabajo coordinado de las diferentes empresas operadoras e instituciones encargadas del transporte público en la ciudad. Este tipo de integración es fundamental para el proceso de unificación del sistema y para alcanzar los otros tipos de integración (Goncalves, 1990). Sin embargo, es el tipo de integración más complejo, ya que requiere de la organización de diferentes actores con intereses particulares. Por su parte, integración operacional, hace referencia a la organización de rutas y líneas, diseño de sistemas tarifarios integrados y aplicación de tecnologías para la integración horaria e informativa de los sistemas de transporte. Finalmente, la integración física, hace referencia a la unificación de los aspectos físicos que conforman el sistema de transporte y comprende la estandarización de los vehículos, de los equipos de recolección de tarifas, de las señalizaciones y del sistema de paradas. También prevé la construcción de instalaciones que faciliten el movimiento peatonal y otros equipamientos de integración tales como estacionamientos para automóviles y bicicletas; y, de particular interés para este artículo, la construcción de estaciones intermodales.

Las estaciones intermodales son definidas como espacios o áreas destinadas "permanentemente a facilitar el intercambio de pasajeros entre diferentes modos de transporte" (Desiderio, 2004). Cuando son implantadas de manera planificada pueden generar beneficios, tales como: reducción del número de transferencias y de los tiempos de viajes; expansión de la cobertura del sistema de transporte público e incremento de su demanda; promoción de modos no motorizados; desarrollo de los usos del suelo adyacentes a la estación; y optimización de la movilidad suburbana e interurbana, al facilitar el acceso de la población residente en la periferia de la ciudad (Rivasplata, 2001; Pitsiava-Latinopoulou y Iordanopoulos, 2012).

\section{Proceso de implantación de las estaciones intermodales}

Horowitz y Thompson (1994) señalan que la localización de las estaciones intermodales en la ciudad determina en gran medida la calidad y eficiencia del servicio de transporte prestado a los usuarios, razón por la que se recomienda que su desarrollo sea guiado por un proceso de implantación definido adecuadamente. Para lograr la implantación de las estaciones intermodales es necesario cubrir una serie de fases, la primera corresponde a la planificación, dentro de ésta una de las etapas corresponde a la definición de las localizaciones, objeto de este trabajo. A continuación, se detalla la fase de planificación y se mencionan las fases posteriores.

\section{Fase de Planificación}

Es compuesta por las etapas:

Definición conceptual: es la etapa inicial del proceso, en la cual se toman decisiones sobre el número de estaciones que poseerá la red y sus características básicas, tales 
como tamaño y los modos de transporte que estarán integrados. Adicionalmente, se consideran las opciones de financiamiento y de desarrollo integrado, se inicia la evaluación preliminar del impacto ambiental y se elaboran diagramas de las áreas candidatas para el emplazamiento de la estación.

Selección de la localización y diseño físico del sistema: con base en los criterios de localización se identifica la ubicación de cada estación, la viabilidad de las conexiones entre los diferentes modos y las prioridades de acceso a la misma. También se realizan los estudios de demanda y se evalúan las diferentes instalaciones físicas del sistema, necesarias para facilitar el movimiento peatonal.

Plan de integración del sistema de transporte: se realiza en paralelo con la etapa anterior y consiste en la preparación del plan de integración en los aspectos institucionales, operacionales y físicos del sistema de transporte. Debe especificar las alternativas para la adecuación de los operadores de transporte y de los servicios de la estación intermodal.

Diseño preliminar y estudio de impacto ambiental: se realiza el diseño preliminar de las estaciones con un nivel de detalle que permita iniciar la evaluación de los impactos en el ambiente y en la comunidad. Adicionalmente, se analizan los aspectos vinculados a la interacción entre los distintos modos y los servicios vinculados. Finalmente, se da inicio a la participación pública para la recolección de observaciones para el plan.

Discusión y aceptación del Plan: consiste en la evaluación y discusión pública del proyecto. Se evalúan las diferentes opciones a fin de seleccionar la más adecuada y se analiza el diseño preliminar de la estación para ser modificado en caso de ser necesario. Se concluye el estudio de impacto ambiental y es abierto a la opinión pública.

El éxito e implementación del plan dependen ampliamente del consenso que se logre entre los actores afectados, entre los cuales se encuentran la comunidad, técnicos y representantes de sectores públicos y privados. Para ello, todos los actores deben estar involucrados incluso, desde las etapas iniciales del proceso. La falta de acuerdo entre los diferentes actores puede llevar a la reestructuración del plan o a la no construcción de la estación intermodal.

\section{Fases siguientes}

En las fases posteriores se definen el financiamiento, el diseño arquitectónico final, la integración institucional y física del sistema, se realizan las obras de construcción y se inicia la operación.

Tomando en cuenta los altos costos de los sistemas ferroviarios como el metro y los recursos limitados de las empresas de transporte, es un desafío asegurar suficientes recursos financieros para su construcción. Además de los préstamos de entes intergubernamentales, se pueden generar nuevos ingresos a través de distintos instrumentos fiscales, dentro de los cuales uno de los más utilizados es la captación de plusvalía (Suzuki et al., 2014).

Por otro lado, para crear estaciones intermodales atractivas se recomienda un enfoque integrado de los aspectos técnicos y estéticos, es decir, la imbricación entre el diseño en términos del uso de transporte (funcionalidad) y el diseño arquitectónico. De esta manera es posible garantizar que el espacio responda a las necesidades de los usuarios (Desiderio, 2004). Los criterios para el diseño arquitectónico de las estaciones han sido tratados por diferentes autores y oficinas responsables de la gestión del transporte (Mbatta et al., 2008) y deben contemplar la integración física de la estación con otros modos de transporte y con el entorno urbano. 
La integración institucional dependerá de los operadores de transporte que estén involucrados y, como fue mencionado, es un elemento fundamental en el proceso de implantación y posterior operación del sistema.

\section{Identificación y clasificación de los criterios de localización}

\section{Clasificación de los criterios de localización de estaciones intermodales}

Con base en la revisión bibliográfica se identifican una serie de criterios generales que deben ser considerados para la selección de localización de las estaciones intermodales (Horowitz y Thompson, 1994; Rivasplata, 2001; SFCTA, 2011). Las fuentes bibliográficas hacen referencia principalmente a contextos de ciudades norteamericanas y europeas. Por tanto, se considera conveniente evaluar estos criterios en el marco de la realidad de las ciudades latinoamericanas y en particular en el caso del AMC. Vale mencionar que, aunque se reconoce la importancia de tomar en cuenta criterios de tipo económico y financiero, estos no son incluidos en el presente estudio.

Para facilitar la comprensión y evaluación de los criterios, se plantea su clasificación dentro de una estructura compuesta por tres ámbitos de análisis: normativo, metropolitano y local. El ámbito normativo incluye aspectos legales, políticas urbanas y normas dirigidas a la protección del medio físico natural, que deben ser respetadas en la implantación de la estación. El ámbito metropolitano reúne criterios que orientan la ubicación de las estaciones intermodales considerando la estructura y dinámica urbana y la interacción entre los distintos sectores de la ciudad. Finalmente, el ámbito local considera criterios que permiten la identificación de la localización específica de la estación tomando en cuenta las características urbanas del sector. Los criterios de localización, a su vez, pueden componerse por diversos elementos específicos (Caneva, 2012). A continuación, se definen los criterios según la estructura de análisis planteada.

\section{Criterios de localización de estaciones intermodales clasificados por ámbito Ámbito normativo \\ En función de las políticas públicas y normativa vigente.}

Con la aplicación de este criterio se busca que la localización de las estaciones intermodales se realice de acuerdo con lo establecido en la normativa urbanística y ambiental vigente, así como en las políticas de urbanismo y de transporte. Incluye los siguientes elementos.

»Con relación a las políticas de movilidad. La localización de la estación debe estar en sintonía con las políticas de transporte dirigidas al mejoramiento de la movilidad urbana;

»Con relación a las políticas de rehabilitación o preservación urbana. La localización de la estación debe favorecer las políticas dirigidas a la recuperación de espacios públicos deteriorados y a la preservación de áreas de valor histórico, cultural o arquitectónico (Goncalves, 1990; Horowitz y Thompson, 1994).

\section{En función de la normativa de protección del medio físico natural.}

Este criterio está dirigido al cumplimiento de la normativa vigente relacionada con la preservación ambiental, a fin de minimizar el impacto sobre el medio natural y el riesgo asociado a la implantación de la estación. Está compuesto por los siguientes elementos específicos. 
» Con relación a áreas que presenten alta amenaza. Debe evitarse la localización de la estación cercana a áreas naturales con condiciones de alta amenaza, y así prevenir la generación de situaciones de riesgos;

»Con relación a la preservación de áreas naturales. La localización de la estación debe ser distante a cuerpos naturales que constituyan áreas de preservación, según lo establecido en la normativa de vigente (Horowitz y Thompson, 1994).

\section{Ámbito metropolitano \\ Localización en nodos donde se maximice el acceso a las diferentes modalida- des de transporte.}

Se refiere a la localización de las estaciones en aquellos puntos de los corredores a los que sea posible acceder fácilmente mediante diversos modos de transporte, a fin de promover la integración modal, o en los nodos en los cuales ya exista un cruce de líneas o rutas de diferentes modalidades de transporte (puntos de transferencia). Se consideran nodos existentes o potenciales donde confluyan un alto número de pasajeros. De este criterio derivan los elementos siguientes (Horowitz y Thompson, 1994; ITDP, 2014):

»Localización en los principales corredores de transporte público: dentro del ámbito de las redes de transporte público de mayor densidad y con conexión a otros modos;

» Localización en redes viales de alta conectividad: en vías ubicadas en zonas que cuenten con alta conectividad vial.

\section{Localización en líneas de ferrocarril y/o en áreas que correspondan al derecho de vía del mismo.}

Prevé la localización de la estación a lo largo de las líneas existentes o propuestas de los sistemas ferroviarios de la ciudad, especialmente si estos estructuran las rutas de transporte superficial (Horowitz y Thompson, 1994).

\section{Localización en zonas de alta demanda de transporte.}

Hace referencia a la conveniencia de localizar la estación en aquellas áreas que constituyan importantes generadores de viajes, a fin de satisfacer la demanda de transporte y captar la demanda potencial (Rivasplata, 2001; SFCTA, 2011; ITDP, 2014).

\section{Localización estratégica para integrar la circulación regional y local.}

Se refiere a la ubicación de la estación en puntos estratégicos que permitan aprovechar la circulación en los principales corredores regionales e integrarlos con el tránsito local o metropolitana, ya sean sistemas existentes o propuestos (Horowitz y Thompson, 1994; Rivasplata, 2001).

\section{Ámbito local}

\section{Localización en zonas vacantes y edificaciones blandas}

Este criterio tiene por objeto minimizar la necesidad de adquisición forzosa de espacios ocupados y disminuir así los costos relacionados con indemnización a propietarios, demolición y posterior implantación de la estación (Horowitz y Thompson, 1994; ITDP, 2014).

\section{Localización en zonas de usos mixtos}

Se propone la localización de las estaciones en zonas de usos mixtos (residencial, equipamientos, comercios, oficinas), a fin de contribuir con la satisfacción de distintas 
demandas, fortalecer la presencia de actividades e incrementar la seguridad percibida por los usuarios, específicamente de los peatones (Rivasplata, 2001; SFCTA, 2011; ITDP, 2014).

\section{Localización cercana a equipamientos recreacionales e hitos arquitectónicos}

La localización de las estaciones cerca de espacios de esparcimiento y edificaciones relevantes para la ciudad puede contribuir a que las mismas sean identificadas como hitos urbanos, favoreciendo adicionalmente el acceso de los usuarios. Las estaciones también pueden ubicarse dentro o cerca de parques y otro tipo de equipamiento urbano, si la demanda y otras consideraciones así lo justifican (SFCTA, 2011).

\section{Evaluación de los criterios, resultados y propuesta de localización de estaciones en el AMC}

\section{Métodos de evaluación}

Los criterios de localización generales y previamente definidos fueron evaluados a través de dos métodos: una encuesta semi-estructurada y un panel de expertos. La encuesta tuvo como objetivo validar la pertinencia de los criterios generales en el AMC, establecer su relevancia y permitir la inclusión observaciones.

Para determinar el número de expertos a encuestar se toma como referencia el trabajo de varios autores que recomiendan entre 2 y 20 personas como un tamaño adecuado para la aplicación del cuestionario (Gable y Wolf, 1993; Grant y Davis, 1997; McGartland et al., 2003). De acuerdo con esto, se decide aplicar el instrumento a un grupo de 20 expertos, 10 nacionales y 10 internacionales, con amplia experiencia en estudios de transporte en ciudades de América Latina, incluyendo el AMC.

La encuesta se elaboró en un formulario en formato PDF, que podía ser editada en las áreas reservadas para las respuestas, fue enviada a través de correo electrónico y fue respondida por 18 expertos. El cuestionario estuvo estructurado en tres apartados: introducción; definición de los criterios de localización; y evaluación de los criterios. Además, se consultaba si se consideraba que los criterios eran adecuados y aplicables a cualquier área urbana y, específicamente, al AMC.

Con relación a la evaluación, el formulario contaba con una tabla con el listado de todos los criterios, organizados por ámbito, para que cada encuestado indicara su relevancia. La evaluación fue realizada con base en la escala cualitativa: muy relevante, relevante, poco relevante, irrelevante. Para identificar el nivel de relevancia de cada uno de los criterios se realizó la sumatoria de las respuestas generadas para cada escala de valoración del criterio. De esta manera se contabilizaron los datos para todos los criterios en evaluación.

Luego de obtenidos y procesados los resultados, se llevó a cabo un panel de expertos, con el objeto de profundizar en la definición y clasificación de los criterios de localización y, a partir de su aplicación en el AMC, generar una propuesta de localización de estaciones intermodales en esta metrópolis.

La selección de los expertos a integrar el panel se basa en tres criterios que fueron establecidos a partir de la bibliografía consultada (Escobar y Cuervo, 2008; Royal Society of Canada, 2010). El primero, que los participantes fuesen profesionales en las áreas de planificación urbana y del transporte; el segundo que tuvieran más de 10 años 
trabajando en proyectos o investigaciones relacionadas con el Área Metropolitana de Caracas; y el tercero, que estuvieran disponibles para participar en el panel durante el período de la investigación.

Estos criterios permitieron reunir a un grupo diverso de seis expertos compuesto por:

》 Gerente de Planificación de CAMETRO (Metro de Caracas).

»Coordinador del estudio del Plan de Caracas 2020.

" Autoridad de transporte metropolitano en el AMC.

» Profesores universitarios con doctorado.

»Consultor privado.

En la bibliografía disponible no se evidencia un consenso sobre el tamaño más adecuado para un panel de expertos (Avella, 2016; Zartha et al., 2014). Aun así, el número de panelistas se adecúa a lo indicado por autores que realizaron metodologías de evaluación similares o que hicieron recomendaciones al respecto (Kauko and Palmroos, 2014; Rowe y Wright, 2001).

El panel de expertos se estructura en tres fases. En la primera fase se realiza la exposición de los objetivos de la investigación y el proceso metodológico, así como los criterios de localización según la clasificación establecida en etapas anteriores. Además, se presentan gráficamente los resultados de la encuesta semi-estructurada.

En la segunda fase del panel se evalúan los resultados de la encuesta. Adicionalmente, se discute acerca de la justificación de las estaciones intermodales en la ciudad, de su tipología y funciones. Por otra parte, se evalúan nuevas propuestas para criterios de localización con el objetivo de definir su inserción o descarte.

Finalmente, la tercera fase consiste en determinar la localización de las estaciones basado en un análisis comparativo entre la experiencia y opinión de los panelistas, la problemática existente en el AMC y los criterios de localización definidos. A partir de esta discusión fue posible también establecer la jerarquización de los criterios para el área de estudio. Para el ejercicio se utilizó la base cartográfica disponible del AMC, así como tablas e imágenes con información relacionada. En particular, fue de especial relevancia, un plano temático donde se indican las líneas existentes y propuestas del sistema de transporte ferroviario de la ciudad, constituido por la Red Metro de Caracas, Ferrocarril Ezequiel Zamora y BusCaracas, Cabletren Bolivariano y sistemas de Metrocable. A fin de alcanzar los objetivos del panel, se plantea que se realicen las sesiones que fuesen necesarias hasta lograr un acuerdo entre los expertos. Se llevaron a cabo dos sesiones en un mismo día, luego de cumplir con las fases y objetivos planteados.

\section{Resultados de la encuesta}

La mayoría de los expertos consultados (89\%) opina que los criterios evaluados son pertinentes y que deben ser adaptados a las características de cada área urbana a fines de la localización de estaciones intermodales. Otro porcentaje de expertos (17\%) destaca que dichos criterios son aplicables especialmente a grandes ciudades o áreas metropolitanas y/o a centros urbanos que posean sistemas de transporte de larga distancia con dificultades para acceder a todas las zonas de la ciudad. Los resultados obtenidos tienden a otorgar mayor relevancia a los criterios de ámbito metropolitano que a los ámbitos normativo y local.

Considerando que la relevancia de los criterios varía de acuerdo con el área urbana, este estudio se centra en las evaluaciones obtenidas para el caso específico del AMC. A continuación, se presentan los resultados agrupados por ámbito de análisis. 


\section{Ámbito normativo}

Tal como se muestra en la Figura 2, el aspecto que obtiene mayor importancia es "localización en función de las políticas de movilidad", al ser considerado por la mayoría de los expertos como muy relevante; seguido de "localización con relación a áreas que presenten alta amenaza natural". Los criterios "localización en función de las políticas de rehabilitación o preservación urbana" y "localización con relación a la preservación ambiental" son calificados principalmente como relevantes. Finalmente, el criterio en "función de otras restricciones legales" es evaluado como relevante por la mayoría de los expertos, al mismo tiempo que sugirieron especificar la normativa, de tal forma que se facilitara su evaluación.

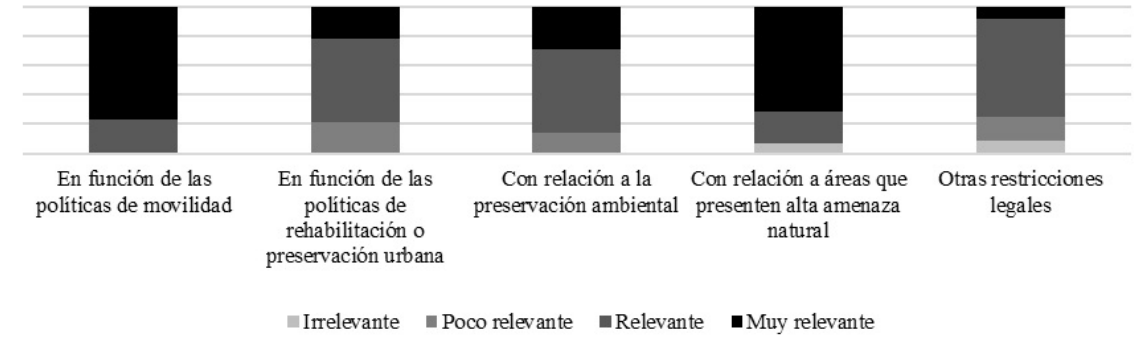

\section{Ámbito metropolitano}

Con relación a los aspectos agrupados en ámbito metropolitano, el criterio "localización en nodos donde se maximice el acceso a las diferentes modalidades de transporte" es evaluado casi de forma unánime como muy relevante y corresponde al aspecto que obtiene la mayor valoración de todos los analizados en el estudio. De forma semejante, el criterio "localización estratégica para integrar la circulación regional y local" es calificado de forma unánime como relevante o muy relevante (ver Figura 3).

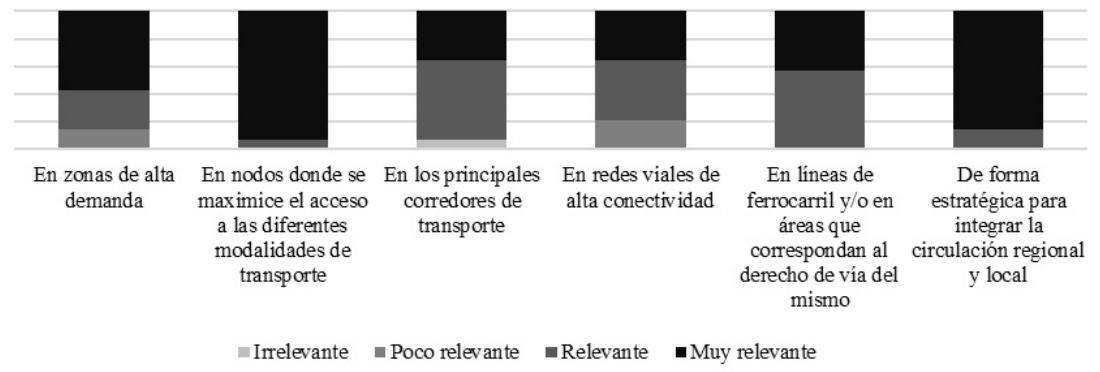

El criterio "localización en zonas de alta demanda de transporte", es valorado mayoritariamente como muy relevante o relevante; mientras que los aspectos localización en los principales corredores de transporte público superficial, localización en líneas de ferrocarril y/o en áreas que correspondan al derecho de vía del mismo y localización en redes viales de alta conectividad, son evaluados principalmente como relevantes.

\section{Ámbito local}

Dentro del ámbito local, el aspecto con mayor relevancia es "localización en zonas de usos mixtos", considerado así por la mayoría de los expertos, aunque las respuestas varían desde irrelevante a muy relevantes (Ver Figura 4). Con relación a la "localización en zonas vacantes y edificaciones blandas" es evaluado mayoritariamente relevante. La "localización cerca de equipamientos recreacionales e hitos arquitectónicos" es evaluada principalmente como poco relevante.
Figura 2. Resultados de evaluación AMC: criterios de localización de ámbito normativo. Fuente: Caneva (2012).
Figura 3. Resultados de evaluación AMC: criterios de localización de ámbito metropolitano. Fuente: Caneva (2012). 


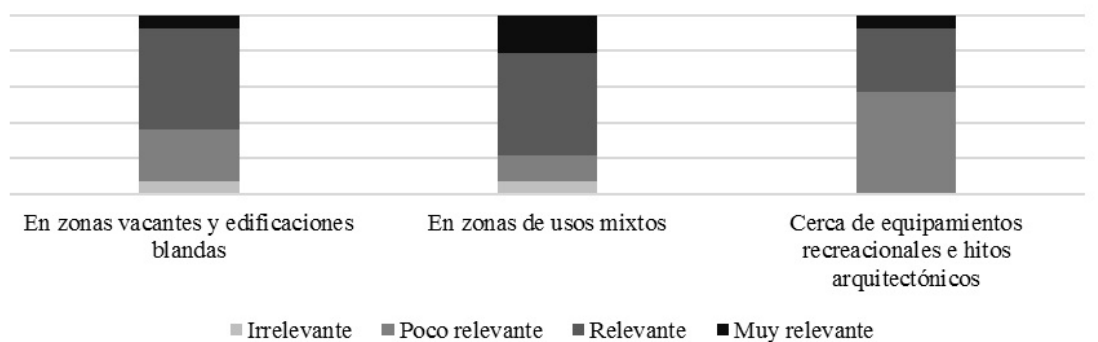

Resultados del panel de expertos

Con base en las discusiones realizadas durante el panel de expertos se adaptan y jerarquizan los criterios de localización aplicables al AMC que fueron evaluados en la encuesta semi-estructurada. La jerarquización se realiza a partir de las opiniones de los expertos participantes, luego de que se realizara un análisis comparativo entre los criterios y la dinámica urbana y del transporte del AMC, logrando un acuerdo entre todos los presentes

Adicionalmente, se define una tipología de estaciones intermodales para el AMC y se realiza una propuesta para la localización de estos equipamientos en la ciudad. Estos resultados se presentan en los apartados siguientes.

\section{Criterios de localización de estaciones intermodales adaptados y jerarquizados para el caso del Área Metropolitana de Caracas}

En el Cuadro 1 se presenta la adaptación y jerarquización de los criterios de localización, agrupados en los tres ámbitos establecidos e incluyendo los elementos asociadas a cada criterio. Algunos de los criterios cambiaron su denominación como consecuencia de la evaluación de los expertos y su adaptación al área de estudio.
Figura 4. Resultados de evaluación AMC: criterios de localización de ámbito local. Fuente: Caneva (2012).

Cuadro 1. Jerarquización de los criterios de localización aplicables al AMC. Fuente: elaboración propia

\begin{tabular}{|l|l|l|}
\hline Ámbito Normativo & Ámbito Metropolitano & Ámbito Local \\
\hline $\begin{array}{l}\text { 1. Localización en función de las } \\
\text { políticas de movilidad. }\end{array}$ & $\begin{array}{l}\text { 1. Localización en nodos donde se } \\
\text { maximice el acceso a las diferentes } \\
\text { modalidades de transporte. }\end{array}$ & $\begin{array}{l}\text { 1. Localización en zonas de usos } \\
\text { mixtos. }\end{array}$ \\
\hline $\begin{array}{l}\text { 2. Localización con relación a áreas } \\
\text { que presenten alta amenaza natural. }\end{array}$ & $\begin{array}{l}\text { 1.1. Localización en los principales } \\
\text { corredores de transporte público } \\
\text { superficial. }\end{array}$ & $\begin{array}{l}\text { 2. Localización en zonas vacantes y } \\
\text { edificaciones blandas. }\end{array}$ \\
\hline $\begin{array}{l}\text { 3. Localización en función de otras } \\
\text { restricciones legales. }\end{array}$ & $\begin{array}{l}\text { 1.2. Localización en redes viales de } \\
\text { alta conectividad. }\end{array}$ & $\begin{array}{l}\text { 3. Localización cerca de } \\
\text { equipamientos recreacionales e hitos } \\
\text { arquitectónicos. }\end{array}$ \\
\hline $\begin{array}{l}\text { 3.1. Localización en función de la } \\
\text { aceptación de la población vecina. }\end{array}$ & $\begin{array}{l}\text { 2. Localización integrada con el } \\
\text { sistema Metro de Caracas. }\end{array}$ & \\
\hline $\begin{array}{l}\text { 4. Localización con relación a la } \\
\text { preservación ambiental. }\end{array}$ & $\begin{array}{l}\text { 3. Localización estratégica para } \\
\text { integrar la circulación regional y local. }\end{array}$ & \\
\hline $\begin{array}{l}\text { 5. Localización en función de } \\
\text { las políticas de rehabilitación o } \\
\text { preservación urbana. }\end{array}$ & $\begin{array}{l}\text { 4. Localización en zonas de altas } \\
\text { demanda de transporte. }\end{array}$ & \\
\hline
\end{tabular}




\section{Implantación y tipología de estaciones intermodales en el Área Metropolitana de Caracas}

Teniendo como referencia las discusiones generadas durante en el panel de expertos, es recomendable que la ubicación de las estaciones se guíe por el proceso de implantación de Horowitz y Thompson (1994) expuesto en apartados anteriores y que, antes de iniciar este proceso, se evalúe si la ciudad requiere de una estación o de un sistema de estaciones intermodales.

Luego de evaluar las condiciones del AMC en cuanto a ciudad policéntrica, a su necesidad de integración con el resto de la Región Metropolitana y a la diversidad de problemas asociados a la inadecuada integración de los modos de transporte, se considera conveniente el desarrollo de una red de estaciones intermodales con funciones diferentes.

Tomando en cuenta las características urbanas y del sistema de transporte, los requerimientos tecnológicos y de espacio de los modos que se pretenden integran a la red, se define la siguiente tipología de estaciones intermodales a tres niveles para el AMC: centrales; semi-periféricas; y semi-periféricas de segundo orden (Caneva, 2012).

\section{Estaciones centrales}

Ubicadas en el eje central de la ciudad, la principal función de estas estaciones es integrar las rutas urbanas alimentadoras con el sistema Metro y, a través de éste, conectar con otras zonas del eje central. Adicionalmente, puede incorporar puntos terminales de rutas o líneas de transporte que tengan origen en las estaciones semi-periféricas. No se recomienda que posean patios donde las unidades de transporte deban permanecer por largos períodos.

\section{Estaciones semi-periféricas}

Localizadas en los límites de la ciudad, la principal función de las estaciones semiperiféricas es albergar rutas alimentadoras provenientes de zonas suburbanas e interurbanas e integrarlas con el sistema de transporte masivo para conectar con el resto de la ciudad. Al igual que las terminales interurbanas de transporte, es adecuado que estas estaciones intermodales se localicen cerca de vías expresas y arteriales, ya que proporcionan acceso directo a otras áreas urbanas sin generar tráfico de paso en el centro de la ciudad (Ocaña y Gómez, 2016).

Estas estaciones podrán reunir rutas alimentadoras urbanas que operen en las cercanías. A diferencia de las estaciones intermodales centrales, las semi-periféricas constituirán terminales de transporte para la mayoría de las rutas asociadas, pudiendo poseer patios y otros componentes. Éstas serán más propensas a la localización de amenidades en sus áreas internas, tales como comercios y otros servicios.

\section{Estaciones semi-periféricas de segundo orden}

Localizadas en los límites del AMC, estas estaciones presentan funciones semejantes a las periféricas con una jerarquía menor, albergan tránsito urbano y suburbano y cuentan con escalas espaciales similares a las estaciones centrales.

\section{Propuesta de localización de estaciones intermodales en el Área Metropolitana de Caracas}

La siguiente propuesta de localización de estaciones intermodales se obtiene de la aplicación de los criterios de ámbito normativo y metropolitano, tomando en cuenta las características de la ciudad, así como los grandes proyectos de transporte planteados para el AMC. 


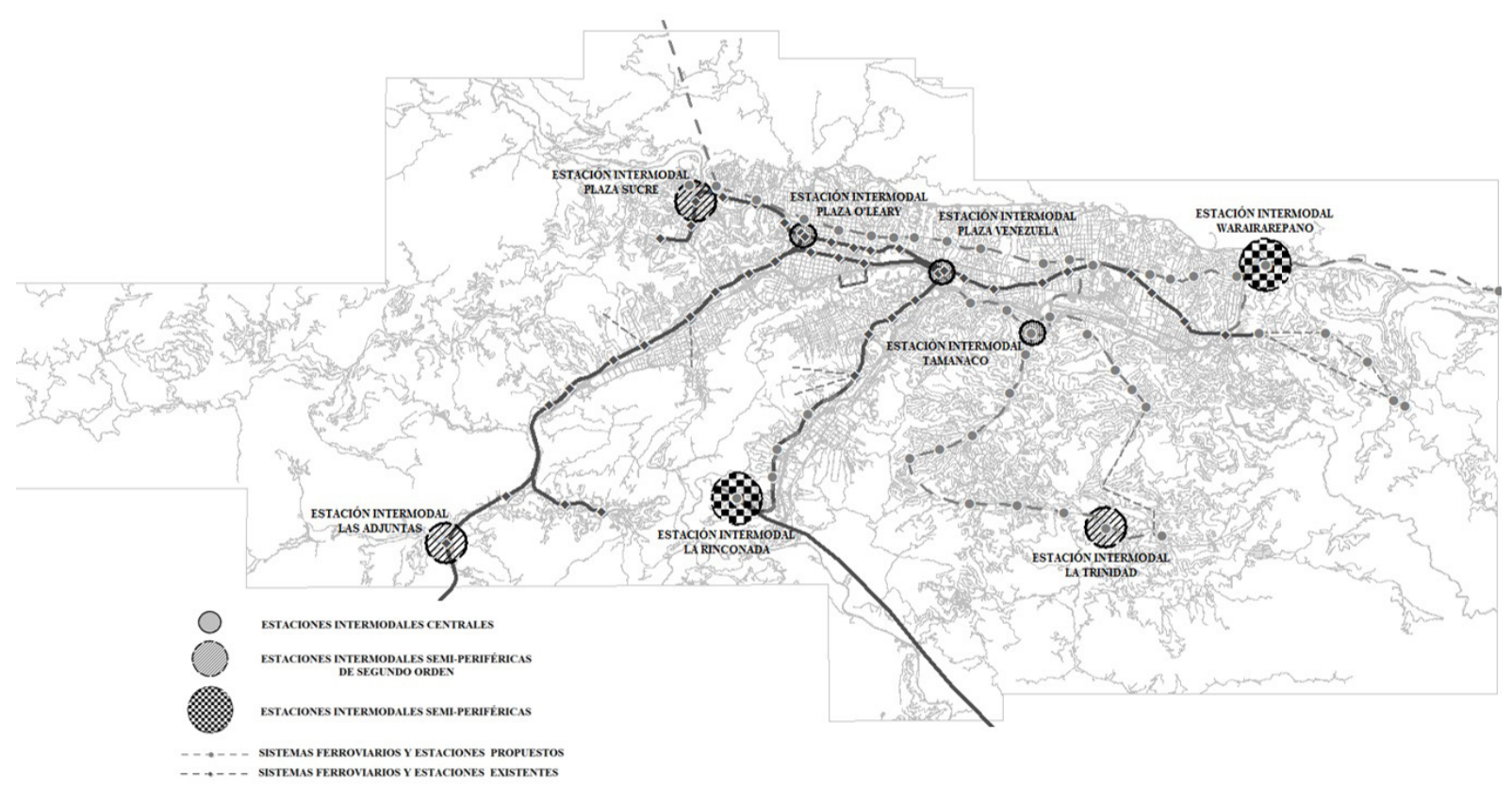

En el desarrollo del panel de expertos se establece que las estaciones deben coincidir con aquellas de la red Metro de Caracas, cumpliendo con el criterio "localización en líneas de ferrocarril y/o en áreas que correspondan al derecho de vía del mismo", el cual se adapta para el caso del AMC como "localización integrada con el sistema Metro".

Figura 5. Propuesta de localización de estaciones intermodales para el AMC. Fuente: Caneva (2012).

Las estaciones son clasificadas según la tipología definida en el apartado anterior. De esta manera, la propuesta contempla el desarrollo de dos estaciones semi-periféricas, tres semi-periféricas de segundo orden y tres centrales (ver Figura 5). A los fines de este artículo, se resumen las propuestas de tres estaciones intermodales, una de cada tipología mencionada (ver Cuadro 2, Cuadro 3 y Cuadro 4).

Cuadro 2. Localización de estaciones intermodales centrales: propuesta estación intermodal Plaza Venezuela. Fuente: Elaboración propia

\begin{tabular}{|l|l|}
\hline Tipo de estación & Intermodal central \\
\hline Características de la zona & $\begin{array}{l}\text { Punto de confluencia de diversas rutas y líneas de transporte. } \\
\text { Cercana a la Autopista Francisco Fajardo y a la Avenida Libertador. } \\
\text { Caracterizada por alta congestión vial y deterioro del espacio } \\
\text { público. } \\
\text { Cercana a diversos centros generadores de viajes como la } \\
\text { Universidad Central de Venezuela, Torre Polar, SENIAT y locales } \\
\text { comerciales. } \\
\text { Disponibilidad de espacio en los terrenos de Zona Rental. }\end{array}$ \\
\hline Justificación: Criterios de localización utilizados & $\begin{array}{l}\text { Localización nodos donde se maximice el acceso a las diferentes } \\
\text { modalidades de transporte. } \\
\text { Localización en redes viales de alta conectividad. } \\
\text { Localización en los principales corredores de transporte público. } \\
\text { Localización en zonas de alta demanda de transporte. } \\
\text { Localización en zonas vacantes y edificaciones blandas. }\end{array}$ \\
\hline Modos de transporte a integrar & $\begin{array}{l}\text { Sistema Metro de Caracas (líneas 1, 3y 5). } \\
\text { Sistemas de transporte público superficial (buses y minibuses). } \\
\text { Sistema Metrobús. }\end{array}$ \\
\hline Tránsito propuesto & Urbano \\
\hline
\end{tabular}


Cuadro 3. Localización de estaciones intermodales semi - periféricas de segundo orden: propuesta estación intermodal La Trinidad. Fuente: Elaboración propia

\begin{tabular}{|l|l|}
\hline Tipo de estación & Intermodal semi - periférica de segundo orden \\
\hline Características de la zona & $\begin{array}{l}\text { Constituirá un importante nodo de transporte al permitir el acceso al } \\
\text { sistema al sector Sureste del AMC. } \\
\text { El Sector Sureste constituye un importante generador de viajes. } \\
\text { Altos niveles de congestión en las vías de acceso. }\end{array}$ \\
\hline Justificación: Criterios de localización utilizados & $\begin{array}{l}\text { Localización nodos donde se maximice el acceso a las diferentes } \\
\text { modalidades de transporte. } \\
\text { Localización en redes viales de alta conectividad. } \\
\text { Localización en los principales corredores de transporte público. } \\
\text { Localización en zonas de alta demanda de transporte. }\end{array}$ \\
\hline Modos de transporte & $\begin{array}{l}\text { Metro de Caracas. } \\
\text { Transporte público superficial (buses y minibuses). } \\
\text { Automóvil particular. }\end{array}$ \\
\hline Tránsito propuesto & Urbano \\
\hline Otras características & $\begin{array}{l}\text { Poseerá equipamiento de integración Park and Ride y Kiss and Ride para } \\
\text { facilitar integración del transporte público y privado. }\end{array}$ \\
\hline
\end{tabular}

Cuadro 4. Localización de estaciones intermodales semi - periféricas: propuesta estación intermodal Waraira Repano. Fuente: Elaboración propia

\begin{tabular}{|l|l|}
\hline Tipo de estación & Intermodal semi - periférica \\
\hline Características de la zona & $\begin{array}{l}\text { Nodo estratégico para la conexión con el este del AMC y del país. } \\
\text { Cercana a la Autopista Guarenas - Guatire, principal conexión entre la } \\
\text { región oriental y el AMC. } \\
\text { Rutas de transporte suburbanos provenientes de la subunidad Guarenas } \\
\text { y Guatire. } \\
\text { Cercana a zonas residenciales como Terrazas del Ávila y Petare (sector } \\
\text { informal). }\end{array}$ \\
\hline Justificación: Criterios de localización utilizados & $\begin{array}{l}\text { Localización nodos donde se maximice el acceso a las diferentes } \\
\text { modalidades de transporte. } \\
\text { Localización en redes viales de alta conectividad. } \\
\text { Localización en los principales corredores de transporte público. } \\
\text { Localización en zonas de alta demanda de transporte. } \\
\text { Localización estratégica para integrar la circulación regional y local. }\end{array}$ \\
\hline Modos de transporte & $\begin{array}{l}\text { Metro de Caracas. } \\
\text { Metro Guarenas - Guatire. } \\
\text { Cabletren Bolivariano. } \\
\text { Transporte público superficial (buses y minibuses). } \\
\text { Automóviles particulares y bicicletas. }\end{array}$ \\
\hline Tránsito propuesto & \begin{tabular}{l} 
Urbano, suburbano, interurbano \\
\hline Otras características
\end{tabular} \\
\hline
\end{tabular}

\section{Discusión y análisis de resultados}

Tomando en cuenta que todos los criterios cuentan con algún nivel de relevancia, con base en la evaluación de expertos, se valida la pertinencia de estos para la toma de decisiones en la localización de estaciones intermodales en el AMC.

A través de la encuesta a expertos se realizó una evaluación comparativa entre los ámbitos normativo, metropolitano y local. De esto, resultó que los criterios de ámbito metropolitano poseen mayor relevancia que aquellos de los ámbitos normativo y local. Sin embargo, con base en el posterior análisis y discusión en el panel de expertos, se concluye que el ámbito normativo debe ser aplicado de forma transversal al resto de 


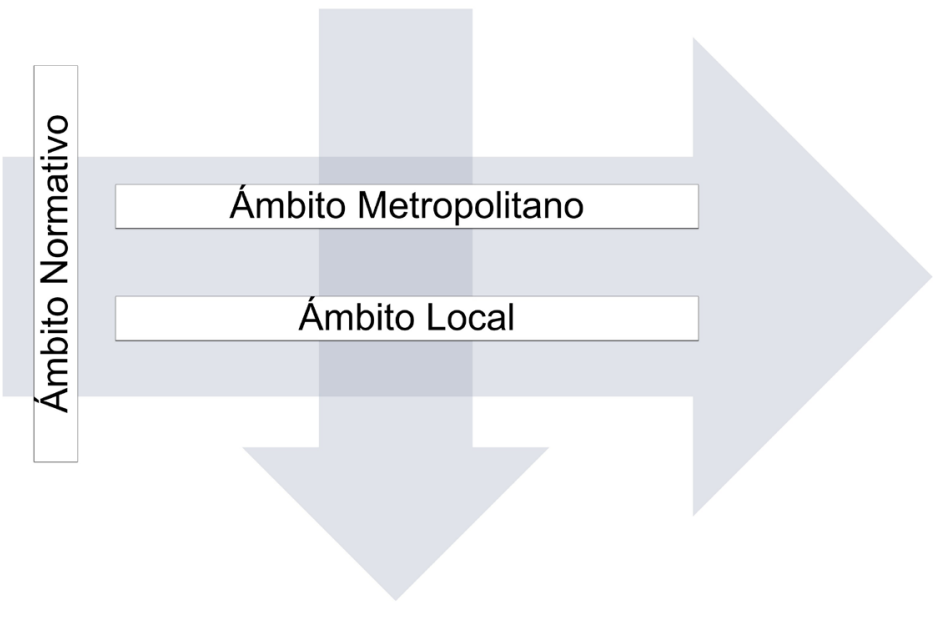

los ámbitos, ya son aspectos de carácter no espacial que constituyen más bien la base legal y política sobre la cual se guía el desarrollo urbano y del sistema de transporte (ver Figura 6).

Por su parte, los criterios de ámbito metropolitano permiten la definición del esquema general de estaciones intermodales tomando en cuenta las condiciones urbanas y de transporte. Contrariamente, los criterios de ámbito local deben ser aplicados posteriormente, al representar aspectos más específicos de la localización.

Los resultados indican que la implantación de estaciones intermodales debe enmarcarse las políticas de movilidad y así coadyuvar en el logro de los objetivos planteados en los planes de usos del suelo y transporte, siendo este criterio el más relevante entre los normativos. En el AMC, el desarrollo de una política de movilidad sostenible, en la cual se incluya la implantación de estaciones intermodales dentro de un sistema de transporte integrado, generaría diversos beneficios a los usuarios, operadores y sociedad en general.

Se concluye que la implantación de estaciones intermodales no tiene el potencial de promover políticas de rehabilitación o preservación urbana en el AMC, ya que la normativa de preservación está desactualizada y los proyectos de recuperación y creación de espacios son generados de forma aislada por distintas autoridades locales y nacionales. Sin embargo, si se realizan acciones conjuntas entre la implantación de la estación y programas de rehabilitación, es posible favorecer estos procesos urbanos y potenciar resultados positivos.

Por otro lado, la consideración de las características del medio físico natural en la implantación de una edificación o equipamiento es un aspecto relevante ya que el AMC es propensa a la ocurrencia de eventos naturales tales como deslizamientos de las laderas, inundaciones y movimientos sísmicos. En consecuencia, las estaciones deben localizarse distantes de las áreas de amenaza en cuanto constituyen equipamientos estratégicos para la ciudad y, de no ser posible, el riesgo potencial debe ser mitigado mediante la aplicación de tecnologías adecuadas para ello.

En general, la relevancia de los criterios de ámbito normativo se ve afectada por la desactualización de la normativa vigente y la falta de coordinación institucional para la generación de planes de desarrollo urbano y del transporte. Esto se refleja en la evaluación realizada por los expertos, quienes opinan que la normativa y zonificación vigente en el AMC debe ser actualizada para garantizar la adecuada implantación de estaciones intermodales.
Figura 6. Relación entre los distintos ámbitos. Fuente: Caneva (2012). 
Con relación al ámbito metropolitano, la localización de la estación en nodos donde se maximice el acceso a distintas modalidades de transporte es un criterio clave y el más relevante entre todos los analizados. Este aspecto se refiere a la ubicación y condiciones necesarias para que la estación efectivamente permita los intercambios entre los distintos sistemas de transporte. Los elementos localización "en los principales corredores de transporte público superficial", "en redes viales de alta conectividad" y "en líneas de ferrocarril y/o en áreas que correspondan al derecho de vía del mismo", son aspectos que contribuyen a maximizar el acceso y, por tanto, están fuertemente relacionados con el primero. El criterio "localización en líneas de ferrocarril" resultó relevante en el AMC por la condición del Metro como eje estructurante del resto de los sistemas de transporte que operan en la ciudad. En la propuesta de estaciones para esta ciudad se establece que las estaciones intermodales coincidan con las del sistema Metro.

Con relación a la ubicación en "redes de alta conectividad", aun cuando este criterio no resulta entre los más relevantes, constituye un factor a considerar por los bajos niveles de conectividad que existen en la red vial del AMC, sobre todo en sentido Norte-Sur.

La localización estratégica para integrar la circulación regional y local, criterio evaluado como relevante por los expertos, debe ser tomado en cuenta principalmente en la evaluación de la localización de estaciones que tienen como función la integración de rutas de transporte urbanas con suburbanas e interurbanas. Dichas estaciones son representadas en la propuesta para el AMC por las estaciones semi-periféricas.

La localización de la estación en zonas de alta demanda en el AMC se considera relevante, porque de esta manera se garantiza la presencia de usuarios potenciales para el transporte público. Esto resulta coherente con los diferentes estudios que han destacado la estrecha relación entre la densidad poblacional y el incremento en el uso del transporte público y modos no motorizados en otras ciudades del mundo (Cervero y Guerra, 2011; UN-HABITAT, 2013).

Particularmente, la localización de estos equipamientos cerca de sectores populares de alta densidad incrementaría potencialmente el uso de las estaciones y de los sistemas integrados en ellas, ya que la población de bajos y medianos ingresos en el AMC se asocia a un mayor uso del transporte público cuando se garantizan condiciones adecuadas de acceso (Caneva, 2016). De esta manera se ampliarían las oportunidades de movilidad para los sectores menos privilegiados de la población caraqueña.

No obstante, la presencia de potenciales usuarios no es una condición suficiente para justificar la implantación de una estación intermodal, ya que deben existir otras condiciones como la posibilidad de conexión con varias modalidades de transporte.

Dentro del ámbito local, el criterio de localización "en zonas de usos mixtos" se considera el aspecto de mayor relevancia en la medida que garantiza mayor intensidad de actividades urbanas generadoras de viajes que favorecen un mayor uso del transporte público y de la estación intermodal (UN-HABITAT, 2013). La localización en "zonas vacantes o con presencia de edificaciones blandas", se considera relevante debido a la poca disponibilidad de espacios vacantes y a las altas densidades edificatorias en el centro del AMC.

\section{Conclusiones}

La localización de estaciones intermodales debe responder a criterios que satisfagan las demandas de transporte de la población y que contribuyan a potenciar los impactos positivos, garantizando que su implantación esté en sintonía con el entorno físico 
natural y construido, la dinámica urbana y los requerimientos espaciales y tecnológicos de los modos de transportes asociados.

Partiendo de esta consideración, se han definido una serie de criterios de localización agrupados en los ámbitos normativo, metropolitano y local que, de acuerdo con los resultados obtenidos, se consideran adecuados y aplicables al Área de Metropolitana de Caracas.

Se propone que los criterios de ámbito normativo sean de aplicación transversal al resto de los criterios, por constituir la base legal para la implantación de cualquier equipamiento en la ciudad. La efectiva aplicación de estos criterios depende de la actualización de la normativa vigente en el AMC.

Los criterios de ámbito metropolitano de mayor relevancia para el AMC son: "localización en nodos donde se maximice el acceso a las diferentes modalidades de transporte" (ámbito metropolitano); "de forma estratégica para integrar la circulación regional y local" (ámbito metropolitano); y "localización en función de las políticas de movilidad" (ámbito normativo).

Se concluye que el AMC cuenta con las condiciones que justifican la implantación de una red de estaciones intermodales que contribuya con la integración de sus distintos modos de transporte, en el marco de las políticas de movilidad sostenible. Así, con la aplicación de los criterios establecidos y la definición de una tipología de estaciones intermodales, se diseña una propuesta de localización de ocho estaciones intermodales con diferentes funciones, que integran las distintas modalidades de transporte presentes en la Región Metropolitana de Caracas.

\section{Recomendaciones}

De acuerdo con los temas tratados en esta investigación se recomienda:

» Adaptar investigaciones y políticas de transporte futuras a los resultados de la encuesta Origen-Destino que se elabora en 2018 para la Región Metropolitana de Caracas, una vez los resultados sean procesados y publicados.

" Desarrollar proyectos dirigidos a la creación de un sistema integrado de transporte, que incluya la integración institucional, operacional y física.

" Profundizar el estudio de localización de estaciones intermodales a través de mesas de trabajo en las cuales participen los diferentes actores de la ciudad (instituciones, operadores, usuarios, sociedad en general).

»Desarrollar planes de movilidad integrados (intermunicipales) que promuevan la integración de los modos de transporte, un mayor uso de modos alternativos (como las bicicletas) y la implantación de estaciones intermodales en el AMC. 


\section{Bibliografía}

»Alcaldía Metropolitana de Caracas (2012). Avances del Plan Estratégico Caracas Metropolitana 2020. Caracas: Autor.

»Avella, J. (2016). Delphi panels: Research design, procedures, advantages, and challenges. International Journal of Doctoral Studies, 11, 305-321.

"Banco de Desarrollo de América Latina (2011). Desarrollo urbano y movilidad en América Latina. Panamá: Autor.

»Barriga Dall'orto-Somelca (200o). Estudio de Infraestructura Vial y Circulación. Informe Fase II. Sin publicar. Caracas: Autor.

»Barrios, S. (2002). La Caracas Metropolitana: De la ciudad compacta a la metrópoli-de-metrópolis. Actas del Seminario Internacional: El Desafío de las Áreas Metropolitanas en un Mundo Globalizado: Una Mirada a Europa y América Latina, pp. 121-138. Barcelona: Institut Català de Cooperación Iberoamericana.

»Burckhart, K. (2007). Análisis comparativo y evaluación cuantitativa de la intermodalidad del tren de alta velocidad. Una perspectiva europea de la interconexión e integración en estaciones ferroviarias de ciudades intermedias. [Tesis doctoral inédita]. Universidad de Lleida, Lleida.

»Caneva, M. (2012). Criterios de localización de estaciones intermodales: un estudio exploratorio en el Área Metropolitana de Caracas. [Tesis de grado inédita]. Universidad Simón Bolívar, Caracas.

"Caneva, M. (2016). Evaluation of Transit Oriented Development principles in the Metropolitan Region of Caracas, Venezuela. [Tesis de maestría inédita]. Universidad de Stuttgart, Alemania.

»Cervero, R., Guerra, E. (2011). Urban densities and transit: a multi-dimensional perspective. Berkley: Institute of Transportation Studies.

»Chaparro, I. (2002). Evaluación del impacto socioeconómico del transporte urbano en la ciudad de Bogotá. El caso del sistema de transporte masivo, Transmilenio. Santiago de Chile: Naciones Unidas.

»Comisión de las Comunidades Europeas (2000). Integrated policy aspects of sustainable mobility. Luxemburgo: Autor.

»Decreto No. 3692 (2005) Del Programa Venezuela Móvil. Gaceta Oficial de la República Bolivariana de Venezuela, 38.205 [Extraordinaria].

»Desiderio, N. (2004). Requirements of users and operators on the design and operation of intermodal interchanges. Recuperado el 25 de Octubre de 2017, de < http://www.verkehr.tu-darmstadt.de/media/verkehr/fgvv/for/publik/Soo7.pdf>

»Escobar, J., Cuervo, Á. (2008). Validez de contenido y juicio de expertos: una aproximación a su utilización. Avances en Medición, 2008 (6), 27-36.

»Flórez, J. (1998). Urban development patterns and their relationship with travel behavior. Policy, Planning and Sustainability. Proceedings of Seminar B, European Transport Conference, 315-330. Association for European Transport by PTRC Educational and Research Services, Ltd. Gran Bretaña.

»Flórez, J. (2000). Incentivando el transporte público. La experiencia de Caracas. Calidad e Innovación en los Transportes. En: Colomer y García (ed.) IV Congreso 
de Ingeniería del Transporte, 2363-2372. Valencia, España.

» Gable, R., Wolf, M. (1993). Instrument development in the affective domain: Measuring attitudes and values in corporate and school settings. Boston: Kluwer Academic Publishers.

》 Goncalves, F. (1990). Lineamientos para la integración del servicio de transporte público de las ciudades venezolanas. [Tesis de maestría inédita]. Universidad Simón Bolívar, Caracas.

" Gonçalves, C., Bandeira, R. (2017). Transporte público em favelas: Análise da percepção da acessibilidade ao teleférico o Alemão. Transportes, 25 (2), 15-28.

»Grant, J., Davis, L. (1997). Selection and use of content experts for instrument development. Research in Nursing \& Health, 20, 269-274.

" Horowitz, A., Thompson, N. (1994). Evaluation of Intermodal Passenger Transfer Facilities. Washington D.C.: Federal Highway Administration.

"Institute for Transportation and Development Policy [ITDP] (2014). TOD standard. New York: Autor.

"Instituto de Estudios Regionales y Urbanos [IERU] (2008) Hacia un acuerdo para mejorar la movilidad en la Región Metropolitana de Caracas. Caracas: Cámara Venezolana de la Construcción.

»Instituto Metropolitano de Transporte [INMETRA] (2005). Planificación y Diseño de una Red Integrada de Transporte Público para el Área Metropolitana de Caracas. Informe: Fase I - Estudio de Movilidad. Alcaldía del Distrito Metropolitano de Caracas: sin publicar.

»Instituto Nacional de Estadística (INE) (2011). XIII Censo General de Población y Vivienda. Venezuela: Autor.

» Kauko, K., Palmroos, P. (2014). The Delphi method in forecasting financial markets-An experimental study. International Journal of Forecasting, 30 (2014), 313-327.

»Llanos, D., Martínez, R. (2014). La planificación urbana en la ciudad de Caracas, Venezuela (1936-2013): En búsqueda de la modernidad perdida. En: Goycoolea, R. (editor). Modernidades ignoradas. Indagaciones sobre arquitectos y obras (casi) desconocidas de la arquitectura moderna. México, Red Nacional de Investigación Urbana, 41-45

"Leclerc, S. (2002). Organización del Transporte Público en las Áreas Metropolitanas Europeas. Recuperado el 25 de Octubre de 2017, de $<$ http://www.madrid.org/cs/BlobServer?blobcol=urldata\&blobtable=MungoB lobs\&blobheadervalue1=filename\%3Do1-Traduccion\%2520de\%2520PaperIEC LER.pdf\&blobkey=id\&blobheadername1=Content-Disposition\&blobwhere $=11$ 81214735637\&blobheader=application\%2Fpdf >

» Lizarraga, C.(2012). Expansión metropolitana y movilidad: el caso de Caracas. EURE, 38 (113), 99-125.

" Mbatta, G., Sando, T., Moses, R. (2008). Developing transit station design criteria with focus on intermodal connectivity. Journal of the Transportation Research Forum, 47 (3), 77-92.

»Mello, C., Rojas, F. (2005). El transporte público colectivo en Curitiba y Bogotá. Revista de Ingeniería, 21 (2005), 106-115.

» Mcgartland, D., Berg-Weger, M., Tebb, S., Lee, S., Rauch, S. (2003). Objectifying content validity: Conducting a content validity study in social work research. 
Social Work Research, 27 (2), 94-104.

»Mundó, J. (2002). El transporte colectivo urbano: aplicación del enfoque de sistemas para un mejor servicio. Fermentum, 12 (34), 285-302.

» Ocaña, R., Gómez, A. (2016). Metodología para evaluación de localización de terminales interurbanos. Portafolio, 2 (34), 49-58.

» Olvera, L., Guézéré, A., Plat, D., Pochet, P. (2015). Improvising intermodality and multimodality. Empirical findings for Lomé, Togo. Case Studies on Transport Policy, 3 (4), 459-467.

» Organisation for Economic Co-Operation and Development [OECD] (2002). Guidelines towards Environmentally Sustainable Transport. Paris: Autor.

»Pitsiava-Latinopoulou, M., lordanopoulos, P. (2012). Intermodal Passengers Terminals: Design standards for better level of service. Procedia - Social and Behavioral Sciences, 48 (2012), 3297-3306.

"Rivasplata, C. (2001). Intermodal transport centres: towards establishing criteria. 2oth South African Conference. Meeting the transport challenges in Southern Africa. Pretoria, Document Transformation Technologies.

»Rojas, Z., Solórzano, J. (2014). Propuesta de localización, requerimientos de intercambiadores modales y estacionamientos disuasorios. [Informe de pasantía inédito]. Universidad Simón Bolívar, Caracas.

"Rowe, G., Wright, G. (2001). Expert opinions in forecasting: the role of the Delphi technique. En: Principles of forecasting, Armstrong, J. (editor), Boston, Kluwer Academic, 125-144.

» Royal Society of Canada (2010). Expert Panels: Manual of Procedural Guidelines. Canada: Autor.

"San Francisco County Transportation Authority [SFCTA] (2011). Bayshore Intermodal Station Access Study. California: Autor.

"Suzuki, H., Cervero, R., Luchi, K. (2014). Transformando las ciudades con el transporte público. Integración del transporte público y el uso del suelo para un desarrollo urbano sostenible. Banco Mundial y Universidad de Bogotá: Washington D.C. y Bogotá.

»Szyliowicz, J. (2003). Decision-making, intermodal transportation, and sustainable mobility: towards a new paradigm. International Social Science Journal, 55 (176), 185-197.

»UN-HABITAT (2013) Urban planning for city leaders. Nairobi: Autor.

»Unión Iberoamericana de Municipalistas [UIAM] (2011). Sistematización de la experiencia, Plan de Movilidad Urbana - Curitiba, Brasil. Recuperado el 12 de Julio del 2012, <http://www.urb-al3.eu/uploads/urbsocialdocumentos/estudio_ de_caso_curitiba.pdf>.

»Urdaneta, C. (2013). La gestión urbana del Área Metropolitana de Caracas. Caracas: Instituto Latinoamericano de Investigaciones Sociales (ILDIS).

"Zartha, J., Montes, J., Toro, I., Villada, H. (2014). Método Delphi - Propuesta para el cálculo del número de expertos en un estudio Delphi sobre empaques biodegradables al 2032. Espacios, 35 (13), 10. 


\section{Marjely Caneva Rodríguez / canevamarjely@gmail.com}

Urbanista (Universidad Simón Bolívar, 2012) y MSc. en Planificación de Infraestructuras (Universidad de Stuttgart, 2016). Desde el año 2013 hasta el año 2014 se desempeñó como Urbanista en la empresa Modelistica - Venezuela. Principal contribución en los proyectos de: "Diseño conceptual de corredores de BRT en el Municipio Libertador de Caracas" y "Estudio para el planteamiento conceptual, accesibilidad vial del nuevo Terminal de Transporte Público en el sector La Rinconada”.

\section{Josefina Flórez-Díaz / jflorez@usb.ve}

Urbanista (Universidad Simón Bolívar- USB, 1986) y Doctora en Transporte y Territorio (Universidad Politécnica de Cataluña, 1998). Profesora Asociada a Dedicación Exclusiva del Departamento de Planificación Urbana de la USB desde 1997. Líneas de investigación: Movilidad Sostenible, Transporte no Motorizado, Dinámica Urbana. http://www.pl.usb.ve/es/node/124 